\title{
Resonance production in Pomeron-Pomeron collisions at the LHC
}

\section{Rainer Schicker*}

Physikalisches Institut, Im Neuenheimer Feld 226, Heidelberg University, 69120 Heidelberg, Germany

E-mail: schickerephysi.uni-heidelberg.de

\section{Roberto Fiore}

Department of Physics, University of Calabria, National Institute of Nuclear Physics, I-87036 Arcavacata di Rende, Cosenza, Italy

E-mail: fioredcs.infn.it

\section{Laszlo Jenkovszky}

Bogolyubov Institute of Theoretical Physics (BITP), Ukrainian National Academy of Sciences, 14-b, Metrologicheskaya str., Kiev, 03680, Ukraine

E-mail: jenkabitp.kiev.ua

A model for Pomeron-Pomeron total cross section in the resonance region $\sqrt{M^{2}} \leq 5 \mathrm{GeV}$ is presented. This model is based on Regge poles from the Pomeron and two different $f$ trajectories, and includes the isolated $\mathrm{f}_{0}(500)$ resonance in the region $\sqrt{M^{2}} \lesssim 1 \mathrm{GeV}$. A slowly varying background is included. The presented Pomeron-Pomeron cross section is not directly measurable, but is an essential ingredient for calculating exclusive resonance production at the LHC.

XXIV International Workshop on Deep-Inelastic Scattering and Related Subjects

11-15 April, 2016

DESY Hamburg, Germany

\footnotetext{
* Speaker.
} 


\section{Introduction}

Central production in proton-proton collisions has been studied in the energy range from the ISR at CERN up to the presently highest LHC energies [1]. Ongoing data analysis include data taken by the COMPASS collaboration at the SPS [2], the CDF collaboration at the TEVATRON [3], the STAR collaboration at RHIC [4], and the ALICE and LHCb collaborations at the LHC [5, 6]. The analysis of events recorded by the large and complex detector systems requires the simulation of such events to study the experimental acceptance and efficiency. Much larger data samples are expected in the next few years both at RHIC and at the LHC allowing the study of differential distributions with much improved statistics. The purpose of the ongoing work presented here is the formulation of a Regge pole model for simulating such differential distributions.

\section{Central production}

The study of central production in hadron-hadron collisions is interesting for a variety of reasons. Such events are characterized by a hadronic system formed at mid-rapidity, and by the two very forward scattered protons, or remnants thereof. The rapidity gap between the mid-rapidity system and the forward scattered proton is a distinctive feature of such events. Central production events can hence be tagged by measuring the forward scattered protons and/or by identifying the existence of rapidity gaps. Central production is dominated at high energies by Pomeron-Pomeron exchange. The hadronization of this gluon-dominated environment is expected to produce with increased probability gluon-rich states, glueballs and hybrids. Of particular interest are states of exotic nature, such as tetra-quark $(q \bar{q}+\bar{q} q)$ configurations, or gluonic hybrids $(q \bar{q}+$ gluon $)$.

\section{Central production event topologies}

The production of central events can take place with the protons remaining in the ground state, or with diffractive excitation of one or both of the outgoing protons.
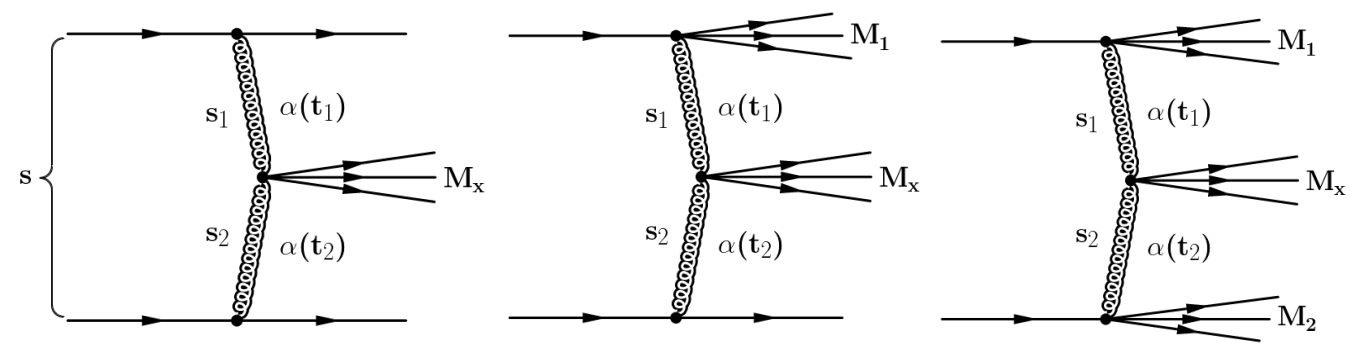

Figure 1: Central production event topologies.

The topologies of central production are shown in Fig. 1. This figure shows central production with the two protons in the ground state on the left, and with one and both protons getting diffractively excited in the middle and on the right, respectively. These reactions take place by the exchange of Regge trajectories $\alpha\left(t_{1}\right)$ and $\alpha\left(t_{2}\right)$ in the central region where a system of mass $\mathrm{M}_{x}$ is produced. The total energy $s$ of the reaction is shared by the subenergies $s_{1}$ and $s_{2}$ associated to 
the trajectories $\alpha\left(t_{1}\right)$ and $\alpha\left(t_{2}\right)$, respectively. The LHC energies of $\sqrt{s}=8$ and $13 \mathrm{TeV}$ are large enough to provide Pomeron dominance. Reggeon exchanges can hence be neglected which was not the case at the energies of previous accelerators.

The main interest in the study presented here is the central part of the diagrams shown in Fig. 1, i.e. Pomeron-Pomeron $(P P)$ scattering producing mesonic states of mass $\mathbf{M}_{x}$. We isolate the Pomeron-Pomeron-meson vertex and calculate the $P P$ total cross section as a function of the centrally produced system of mass $\mathbf{M}_{x}$. The emphasis here is the behaviour in the low mass resonance region where perturbative QCD approaches are not applicable. Instead, similar to [7], we use the pole decomposition of a dual amplitude with relevant direct-channel trajectories $\alpha\left(M^{2}\right)$ for fixed values of Pomeron virtualities, $t_{1}=t_{2}=$ const. Due to Regge factorization, the calculated Pomeron-Pomeron cross section is part of the measurable proton-proton cross section [8].

\section{Dual resonance model of Pomeron-Pomeron scattering}

Most of the existing studies on diffraction dissociation, single, double and central, are done within the framework of the triple Reggeon approach. This formalism is useful beyond the resonance region, but is not valid for the low mass region which is dominated by resonances. A formalism to account for production of resonances was formulated in Ref. [9]. This formalism is based on the idea of duality with a limited number of resonances represented by nonlinear Regge trajectories.

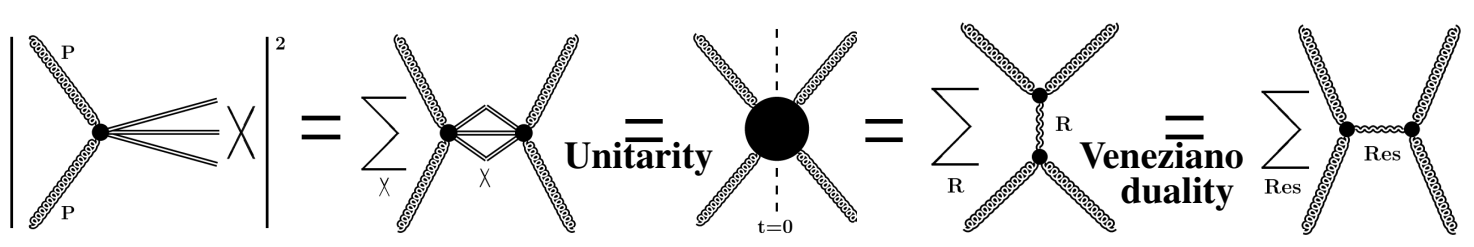

Figure 2: Connection, through unitarity (generalized optical theorem) and Veneziano-duality, between the Pomeron-Pomeron cross section and the sum of direct-channel resonances.

The motivation of this approach consists of using dual amplitudes with Mandelstam analyticity (DAMA), and is shown in Fig. 2. For $s \rightarrow \infty$ and fixed $t$ it is Regge-behaved. Contrary to the Veneziano model, DAMA not only allows for, but rather requires the use of nonlinear complex trajectories which provide the resonance widths via the imaginary part of the trajectory. A finite number of resonances is produced for limited real part of the trajectory.

For our study of central production, the direct-channel pole decomposition of the dual amplitude $A\left(M_{X}^{2}, t\right)$ is relevant. This amplitude receives contributions from different trajectories $\alpha_{i}\left(M_{X}^{2}\right)$, with $\alpha_{i}\left(M_{X}^{2}\right)$ a nonlinear, complex Regge trajectory in the Pomeron-Pomeron system,

$$
A\left(M_{X}^{2}, t\right)=a \sum_{i=f, P} \sum_{J} \frac{\left[f_{i}(t)\right]^{J+2}}{J-\alpha_{i}\left(M_{X}^{2}\right)} .
$$

The pole decomposition of the dual amplitude $A\left(M_{X}^{2}, t\right)$ is shown in Eq. (4.1), with $t$ the squared momentum transfer in the $P P \rightarrow P P$ reaction. The index $i$ sums over the trajectories which contribute to the amplitude. Within each trajectory, the second sum extends over the bound states of spin $J$. The prefactor $a$ in Eq. (4.1) is of numerical value $\mathrm{a}=1 \mathrm{GeV}^{-2}=0.389 \mathrm{mb}$. 
The imaginary part of the amplitude $A\left(M_{X}^{2}, t\right)$ given in Eq. (4.1) is defined by

$$
\mathfrak{I} m A\left(M_{X}^{2}, t\right)=a \sum_{i=f, P} \sum_{J} \frac{\left[f_{i}(t)\right]^{J+2} \mathfrak{I} m \alpha_{i}\left(M_{X}^{2}\right)}{\left(J-\operatorname{Re} \alpha_{i}\left(M_{X}^{2}\right)\right)^{2}+\left(\mathfrak{I} m \alpha_{i}\left(M_{X}^{2}\right)\right)^{2}} .
$$

For the $P P$ total cross section we use the norm

$$
\sigma_{t}^{P P}\left(M_{X}^{2}\right)=\mathfrak{I} m A\left(M_{X}^{2}, t=0\right) .
$$

The Pomeron-Pomeron channel, $P P \rightarrow M_{X}^{2}$, couples to the Pomeron and $f$ channels due to quantum number conservation. For calculating the $P P$ cross section, we therefore take into account the trajectories associated to the $\mathrm{f}_{0}(980)$ and to the $\mathrm{f}_{2}(1270)$ resonance, and the Pomeron trajectory.

\section{Non-linear, complex meson Regge trajectories}

Analytic models of Regge trajectories need to derive the imaginary part of the trajectory from the almost linearly increasing real part. We relate the nearly linear real part of the meson trajectory to its imaginary part by following Ref. [10],

$$
\mathfrak{R} e \alpha(s)=\alpha(0)+\frac{s}{\pi} P V \int_{0}^{\infty} d s^{\prime} \frac{\mathfrak{I} m \alpha\left(s^{\prime}\right)}{s^{\prime}\left(s^{\prime}-s\right)} .
$$

In Eq. 5.1, the dispersion relation connecting the real and imaginary part is shown. The imaginary part of the trajectory is related to the decay width by

$$
\Gamma\left(M_{R}\right)=\frac{\mathfrak{I} m \alpha\left(M_{R}^{2}\right)}{\alpha^{\prime} M_{R}} .
$$

\section{The Regge trajectories}

Apart from the Pomeron trajectory, the direct-channel $f$ trajectory is essential in the PP system. Guided by conservation of quantum numbers, we include two $f$ trajectories, labeled $f_{1}$ and $f_{2}$, with mesons lying on these trajectories as specified in Table 1.

\begin{tabular}{|c|cc|c||c|c|c||}
\hline & $\mathrm{I}^{G}$ & $\mathrm{~J}^{P C}$ & traj. & $\mathrm{M}(\mathrm{GeV})$ & $\mathrm{M}^{2}\left(\mathrm{GeV}^{2}\right)$ & $\Gamma(\mathrm{GeV})$ \\
\hline $\mathrm{f}_{0}(980)$ & $0^{+}$ & $0^{++}$ & $f_{1}$ & $0.990 \pm 0.020$ & $0.980 \pm 0.040$ & $0.070 \pm 0.030$ \\
$\mathrm{f}_{1}(1420)$ & $0^{+}$ & $1^{++}$ & $f_{1}$ & $1.426 \pm 0.001$ & $2.035 \pm 0.003$ & $0.055 \pm 0.003$ \\
$\mathrm{f}_{2}(1810)$ & $0^{+}$ & $2^{++}$ & $f_{1}$ & $1.815 \pm 0.012$ & $3.294 \pm 0.044$ & $0.197 \pm 0.022$ \\
$\mathrm{f}_{4}(2300)$ & $0^{+}$ & $4^{++}$ & $f_{1}$ & $2.320 \pm 0.060$ & $5.382 \pm 0.278$ & $0.250 \pm 0.080$ \\
$\mathrm{f}_{2}(1270)$ & $0^{+}$ & $2^{++}$ & $f_{2}$ & $1.275 \pm 0.001$ & $1.6256 \pm 0.003$ & $0.185 \pm 0.003$ \\
$\mathrm{f}_{4}(2050)$ & $0^{+}$ & $4^{++}$ & $f_{2}$ & $2.018 \pm 0.011$ & $4.0723 \pm 0.044$ & $0.237 \pm 0.018$ \\
$\mathrm{f}_{6}(2510)$ & $0^{+}$ & $6^{++}$ & $f_{2}$ & $2.469 \pm 0.029$ & $6.096 \pm 0.143$ & $0.283 \pm 0.040$ \\
\hline
\end{tabular}

Table 1: Parameters of resonances belonging to the $f_{1}$ and $f_{2}$ trajectories.

The real and imaginary part of the $f_{1}$ and $f_{2}$ trajectories can be derived from the parameters of the f-resonances listed in Table 1, and has explicitely been derived in Ref. [11].

While ordinary meson trajectories can be fitted both in the resonance and scattering region corresponding to positive and negative values of the argument, the parameters of the Pomeron 
trajectory can only be determined in the scattering region $M^{2}<0$. A comprehensive fit to highenergy $p p$ and $p \bar{p}$ of the nonlinear Pomeron trajectory is discussed in Ref.[8]

$$
\alpha_{P}\left(M^{2}\right)=1 .+\varepsilon+\alpha^{\prime} M^{2}-c \sqrt{s_{0}-M^{2}},
$$

with $\varepsilon=0.08, \alpha^{\prime}=0.25 \mathrm{GeV}^{-2}, \mathrm{~s}_{0}$ the two pion threshold $\mathrm{s}_{0}=4 \mathrm{~m}_{\pi}^{2}$, and $\mathrm{c}=\alpha^{\prime} / 10=0.025$.

For consistency with the mesonic trajectories, the linear term in Eq. (6.1) is replaced by a heavy threshold mimicking linear behaviour in the mass region of interest $(\mathrm{M}<5 \mathrm{GeV})$,

$$
\alpha_{P}\left(M^{2}\right)=\alpha_{0}+\alpha_{1}\left(2 m_{\pi}-\sqrt{4 m_{\pi}^{2}-M^{2}}\right)+\alpha_{2}\left(\sqrt{M_{H}^{2}}-\sqrt{M_{H}^{2}-M^{2}}\right)
$$

with $M_{H}$ an effective heavy threshold $M=3.5 \mathrm{GeV}$. The coefficients $\alpha_{0}, \alpha_{1}$ and $\alpha_{2}$ are chosen such that the Pomeron trajectory of Eq. (6.2) has a low energy behaviour as defined by Eq. (6.1).

\section{The $f_{0}(500)$ resonance}

The experimental data on central exclusive pion-pair production measured at the energies of the ISR, RHIC, TEVATRON and the LHC collider all show a broad continuum for pair masses $\mathrm{m}_{\pi^{+} \pi^{-}}<1 \mathrm{GeV} / \mathrm{c}^{2}$. The population of this mass region is attributed to the $f_{0}(500)$. This resonance $f_{0}(500)$ is of prime importance for the understanding of the attractive part of the nucleon-nucleon interaction, as well as for the mechanism of spontaneous breaking of chiral symmetry. In spite of the complexity of the $f_{0}(500)$ resonance, and the controversy on its interpretion and description, we take here the practical but simple-minded approach of a Breit-Wigner resonance [12]

$$
A\left(M^{2}\right)=a \frac{-M_{0} \Gamma}{M^{2}-M_{0}^{2}+i M_{0} \Gamma} .
$$

The Breit-Wigner amplitude of Eq. (7.1) is used below for calculating the contribution of the $f_{0}(500)$ resonance to the Pomeron-Pomeron cross section.

\section{Pomeron-Pomeron total cross section}

The Pomeron-Pomeron cross section is calculated from the imaginary part of the amplitude by use of the optical theorem

$$
\sigma_{t}^{P P}\left(M^{2}\right)=\mathfrak{I} m A\left(M^{2}, t=0\right)=\sum_{i=f, P} \sum_{J} \frac{\left[f_{i}(0)\right]^{J+2} \mathfrak{I} m \alpha_{i}\left(M^{2}\right)}{\left(J-\mathfrak{R} e \alpha_{i}\left(M^{2}\right)\right)^{2}+\left(\mathfrak{I} m \alpha_{i}\left(M^{2}\right)\right)^{2}}
$$

In Eq. (8.1), the index $i$ sums over the trajectories which contribute to the cross section, in our case the $f_{1}, f_{2}$ and the Pomeron trajectory discussed above. Within each trajectory, the summation extends over the bound states of spin $J$ as expressed by the second summation sign. The value $f_{i}(0)=\left.f_{i}(t)\right|_{t=0}$ is not known a priori. The analysis of relative strengths of the states of trajectory $i$ will, however, allow to extract a numerical value for $f_{i}(0)$ from the experimental data.

The Pomeron-Pomeron total cross section is calculated by summing over the contributions discussed above, and is shown in Fig. 3 by the solid black line. The prominent structures seen in the total cross section are labeled by the resonances generating the peaks. The model presented here 


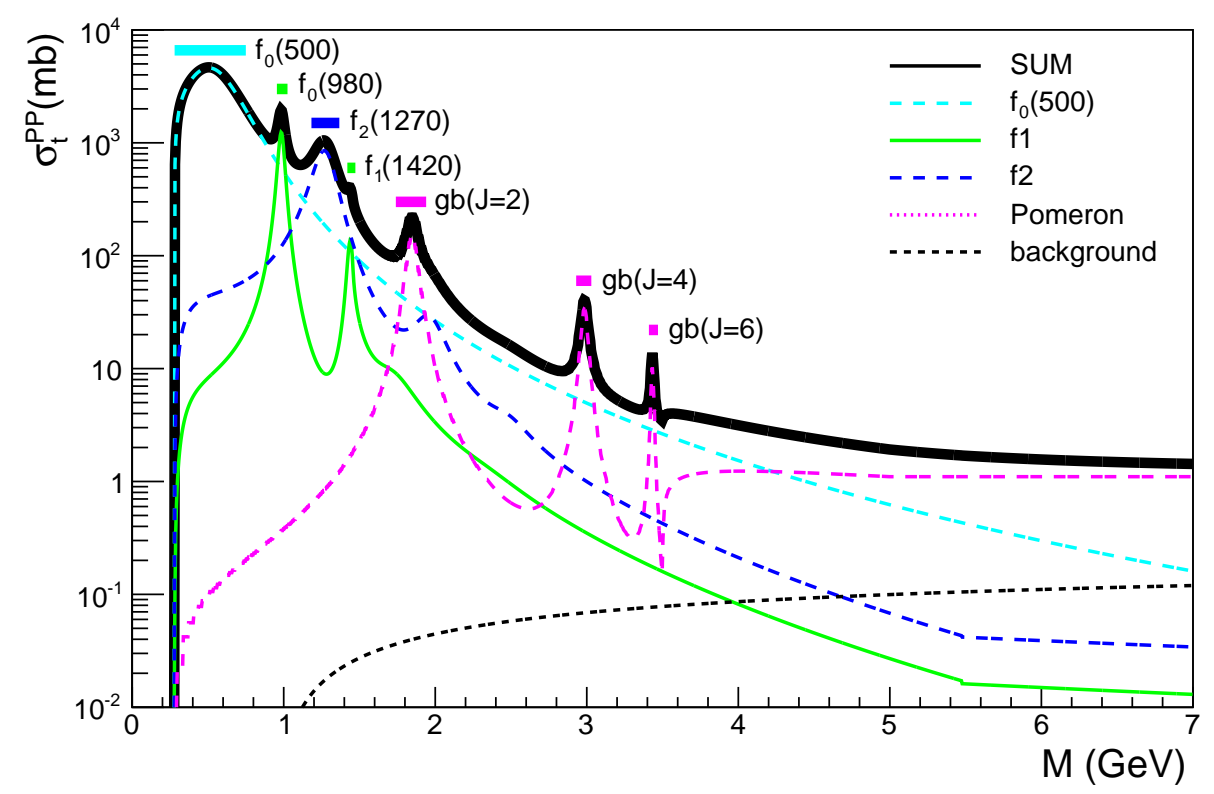

Figure 3: Contributions of the $\mathrm{f}_{0}(500)$ resonance, the $f_{1}, f_{2}$ and the Pomeron trajectory, and of the background to PP total cross section.

does not specify the relative strength of the different contributions shown in Fig. 3. A Partial Wave Analysis of experimental data on central production events will be able to extract the quantum numbers of these resonances, and will hence allow to associate each resonance to its trajectory. The relative strengths of the contributing trajectories need to be taken from the experimental data.

\section{References}

[1] M. Albrow, V. Khoze, Ch. Royon, Special Issue Int. J. Mod. Phys. A29 (2014) 28.

[2] A. Austregesilo, for the COMPASS Coll., Proc. 15th EDS Conference, Sept 2013, arXiv:1310.3190.

[3] M. Albrow, for the CDF Coll., Int. J. Mod.Phys. A29 (2014) 28, 1446009, arXiv:1409.0462.

[4] L. Adamczyk, W. Guryn and J. Turnau, Int. J. Mod.Phys. A29 (2014) 28, 1446010, arXiv:1410.5752.

[5] R. Schicker, Int.J.Mod.Phys. A29 (2014) 28, 1446015, arXiv:1411.1283.

[6] R. McNulty, Int. J. Mod.Phys. A29 (2014) 28, 1446003, arXiv:1409.8113.

[7] L. Jenkovszky, O. Kuprash, J.W. Lamsa, V.K. Magas, and R.Orava, Phys.Rev.D 83 (2011) 056014, arXiv:1011.0664.

[8] L. Jenkovszky, Rivista Nuovo Cim., 10 (1987), p. N12.

[9] R. Fiore, A. Flachi, L. Jenkovszky, A. Lengyel and V. Magas, Phys. Rev. D 69 (2004), 014004, hep-ph/0308178.

[10] R. Fiore, L. Jenkovszky, V. Magas, F. Paccanoni, A. Papa, Eur.Phys.J. A10 (2001) 217-221, hep-ph/0011035.

[11] R. Fiore, L. Jenkovszky, R. Schicker, Eur.Phys.J. C76 (2016) no.1, 38, arXiv:1512.04977.

[12] Review of Particle Physics, Chinese Physics C 38, 9 (2014). 